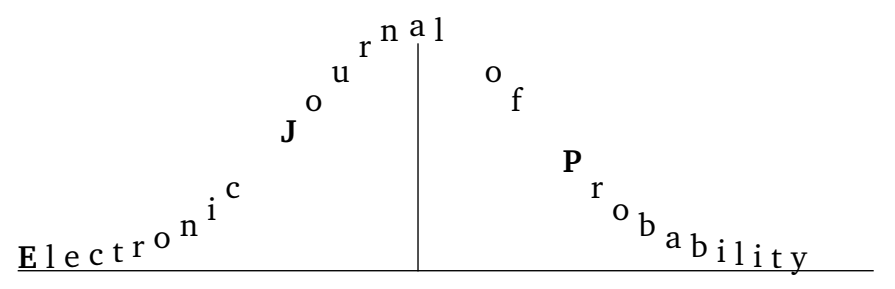

Vol. 14 (2009), Paper no. 20, pages 531-550.

Journal URL

http://www.math.washington.edu/ ejpecp/

\title{
Coarse graining, fractional moments and the critical slope of random copolymers*
}

\author{
Fabio Lucio Toninelli \\ CNRS and ENS Lyon, Laboratoire de Physique, \\ 46 Allée d'Italie, 69364 Lyon, France. \\ E-mail address: fabio-lucio.toninelli@ens-lyon.fr
}

\begin{abstract}
For a much-studied model of random copolymer at a selective interface we prove that the slope of the critical curve in the weak-disorder limit is strictly smaller than 1 , which is the value given by the annealed inequality. The proof is based on a coarse-graining procedure, combined with upper bounds on the fractional moments of the partition function.
\end{abstract}

Key words: Copolymers at Selective Interfaces, Fractional Moment Estimates, Coarse-graining. AMS 2000 Subject Classification: Primary 60K35, 82B44, 60K37.

Submitted to EJP on June 9, 2008, final version accepted January 30, 2009.

\footnotetext{
${ }^{*}$ Work partially supported by ANR, grant POLINTBIO and grant LHMSHE
} 


\section{Introduction}

We consider a model of copolymer at a selective interface introduced in [10], which has attracted much attention among both theoretical physicists and probabilists (we refer to [11] for general references and motivations).

Let $S:=\left\{S_{n}\right\}_{n \geq 0}$ be the symmetric Simple Random Walk on $\mathbb{Z}$ started at $S_{0}=0$, with law $\mathbf{P}^{\mathrm{SRW}}$ such that the increments $\left\{S_{i}-S_{i-1}\right\}_{i \geq 0}$ are IID and $\mathbf{P}^{\mathrm{SRW}}\left(S_{1}= \pm 1\right)=1 / 2$. The partition function of the model of size $N$ is given by

$$
Z_{N, \omega}:=\mathbf{E}^{\mathrm{SRW}}\left[e^{-\lambda \sum_{n=1}^{N}\left(\omega_{n}+h\right)\left(1-\operatorname{sign}\left(S_{n}\right)\right)} \mathbf{1}_{\left\{S_{N}=0\right\}}\right]
$$

where $h \geq 0, \lambda \geq 0$ and $\left\{\omega_{n}\right\}_{n \geq 1}$ is a sequence of IID standard Gaussian random variables (the quenched disorder). We adopt the convention that, if $S_{n}=0$, then $\operatorname{sign}\left(S_{n}\right):=\operatorname{sign}\left(S_{n-1}\right)$. One interprets $\lambda$ as the inverse temperature (or coupling strength) and $h$ as an "asymmetry parameter": if $h>0$, since the $\omega_{n}$ 's are centered, the random walk overall prefers to be in the upper halfplane $(S \geq 0)$. It is known that the model undergoes a delocalization transition: if the asymmetry parameter exceeds a critical value $h_{c}(\lambda)$ then the fraction of "monomers" $S_{n}, n \leq N$, which are in the upper half-plane tends to 1 in the thermodynamic limit $N \rightarrow \infty$ (delocalized phase), while if $h<h_{c}(\lambda)$ then a non-zero fraction of them is in the lower half-plane (localized phase). What mostly attracts attention is the slope, call it $m_{c}$, of the curve $\lambda \mapsto h_{c}(\lambda)$ in the limit $\lambda \searrow 0: m_{c}$ is expected to be a universal quantity, i.e., independent of the details of the law $\mathbf{P}^{\mathrm{SRW}}$ and of the disorder distribution (see next section for a more extended discussion on this point). Already the fact that the limit slope is well-defined and positive is highly non-trivial [5].

Until now, all what was known rigorously about $m_{c}$ is that $2 / 3 \leq m_{c} \leq 1$, but numerically the true value seems to be rather around 0.83 [6]. The upper bound comes simply from annealing, i.e., from Jensen's inequality, as explained in next section. Our main new result is that $m_{c}$ is strictly smaller than 1 . The proof works through a coarse-graining procedure in which one looks at the system on the length-scale $k(\lambda, h)$, given by the inverse of the annealed free energy. The other essential ingredient is a change-of-measure idea to estimate fractional moments of the partition function (this idea was developed in [12] and [7], and used in the context of copolymers in [3]). Coarse-graining schemes, implemented in a way very different from ours, have already played an important role in this and related polymer models; we mention in particular [5], [4] and [1].

\section{The general copolymer model}

As in [3], we consider a more general copolymer model which includes (1.1) as a particular case. Since the critical slope is not proven to exist in this general setting, Theorem 2.1 will involve a limsup instead of a limit.

Consider a renewal process $\tau:=\left\{\tau_{0}, \tau_{1}, \ldots\right\}$ of law $\mathbf{P}$, where $\tau_{0}:=0$ and $\left\{\tau_{i}-\tau_{i-1}\right\}_{i \in \mathbb{N}}$ is an IID sequence of integer-valued random variables. We call $K(n):=\mathbf{P}\left(\tau_{1}=n\right)$ and we assume that $\sum_{n \in \mathbb{N}} K(n)=1$ (the renewal is recurrent) and that $K(\cdot)$ has a power-law tail:

$$
K(n) \stackrel{n \rightarrow \infty}{\sim} \frac{\mathscr{C}_{K}}{n^{1+\alpha}}
$$


with $\alpha>0$ and $\mathscr{C}_{K}>0$. As usual, the notation $A(x) \stackrel{x \rightarrow x_{0}}{\sim} B(x)$ is understood to mean that $\lim _{x \rightarrow x_{0}} A(x) / B(x)=1$.

The copolymer model we are going to define depends on two parameters $\lambda \geq 0$ and $h \geq 0$, and on a sequence $\omega:=\left\{\omega_{1}, \omega_{2}, \ldots\right\}$ of IID standard Gaussian random variables (the quenched disorder), whose law is denoted by $\mathbb{P}$. For a given system size $N \in \mathbb{N}$ and disorder realization $\omega$, we define the partition function $Z_{N, \omega}:=Z_{N, \omega}(\lambda, h)$ as

$$
Z_{N, \omega}:=\mathbf{E}\left[\prod_{j \geq 1: \tau_{j} \leq N} \frac{1+e^{-2 \lambda h\left(\tau_{j}-\tau_{j-1}\right)-2 \lambda \sum_{i=\left(\tau_{j-1}+1\right)}^{\tau_{j}} \omega_{i}}}{2} \mathbf{1}_{\{N \in \tau\}}\right],
$$

where $\mathbf{1}_{\{A\}}$ is the indicator function of the event $A$.

To see that the "standard copolymer model" (1.1) is a particular case of (2.2), let $\tau:=\{n \geq 0 \in$ $\left.\mathbb{N}: S_{n}=0\right\}$ and as a consequence $K(n):=\mathbf{P}^{\mathrm{SRW}}\left(\inf \left\{k>0: S_{k}=0\right\}=n\right)$. It is known that in this case $K(\cdot)$ satisfies (2.1) with $\alpha=1 / 2$, see [9, Ch. III] (the fact that in this case (2.1) holds only for $n \in 2 \mathbb{N}$, while $K(n)=0$ for $n \in 2 \mathbb{N}+1$ due to the periodicity of the simple random walk, entails only elementary modifications in the arguments below). Next, observe that if $s_{i} \in\{-1,+1\}$ denotes the sign of the excursion of $S$ between the successive returns to zero $\tau_{i-1}$ and $\tau_{i}$, under $\mathbf{P}^{S R W}$ the sequence $\left\{s_{i}\right\}_{i \in \mathbb{N}}$ is IID and symmetric (and independent of the sequence $\tau$ ). Therefore, performing the average on the $\left\{s_{i}\right\}_{i}$ in (1.1) one immediately gets (2.2).

The infinite-volume free energy is defined as

$$
\mathrm{F}(\lambda, h):=\lim _{N \rightarrow \infty} \frac{1}{N} \mathbb{E} \log Z_{N, \omega} \geq 0,
$$

where existence of the limit is a consequence of superadditivity of the sequence $\left\{\mathbb{E} \log Z_{N, \omega}\right\}_{N}$ and the inequality $\mathrm{F} \geq 0$ is immediate from

$$
Z_{N, \omega} \geq \frac{K(N)}{2}
$$

which is easily seen inserting in the expectation in right-hand side of (2.2) the indicator function $\mathbf{1}_{\left\{\tau_{1}=N\right\}}$. One usually defines the critical line in the $(\lambda, h)$ plane as

$$
h_{c}(\lambda):=\sup \{h \geq 0: \mathrm{F}(\lambda, h)>0\} .
$$

From Jensen's inequality $\mathbb{E} \log Z_{N, \omega} \leq \log \mathbb{E} Z_{N, \omega}$ (the "annealed bound") one obtains the immediate inequality

$$
h_{c}(\lambda) \leq \lambda
$$

Indeed, one has

$$
\mathbb{E} Z_{N, \omega}=\mathbf{E}\left[\prod_{j \geq 1: \tau_{j} \leq N} \frac{1+e^{2 \lambda(\lambda-h)\left(\tau_{j}-\tau_{j-1}\right)}}{2} \mathbf{1}_{\{N \in \tau\}}\right]
$$

from which it is not difficult to prove that

$$
\lim _{N \rightarrow \infty} \frac{1}{N} \log \mathbb{E} Z_{N, \omega}=2 \lambda(\lambda-h) 1_{\{h<\lambda\}},
$$


and therefore the claim (2.6). For $h \geq \lambda$, (2.8) follows from the fact that the right-hand side of (2.7) is bounded above by 1 , while the left-hand side of (2.8) is always non-negative. For $h<\lambda$, just observe that

$$
\mathbb{E} Z_{N, \omega} \leq \mathbf{E}\left[\prod_{j \geq 1: \tau_{j} \leq N} e^{2 \lambda(\lambda-h)\left(\tau_{j}-\tau_{j-1}\right)} \mathbf{1}_{\{N \in \tau\}}\right]=\mathbf{P}(N \in \tau) e^{2 \lambda(\lambda-h) N}
$$

and that

$$
\mathbb{E} Z_{N, \omega} \geq \mathbf{E}\left[\prod_{j \geq 1: \tau_{j} \leq N} \frac{1+e^{2 \lambda(\lambda-h)\left(\tau_{j}-\tau_{j-1}\right)}}{2} \mathbf{1}_{\left\{\tau_{1}=N\right\}}\right]=K(N) \frac{1+e^{2 \lambda(\lambda-h) N}}{2} .
$$

The limit (2.8) is called annealed free energy.

\subsubsection{What is known about the critical line and its slope at the origin}

The critical point is known to satisfy the bounds

$$
\frac{\lambda}{1+\alpha} \leq h_{c}(\lambda)<\lambda
$$

The upper bound, proven recently in [3, Th. 2.10], says that the annealed inequality (2.6) is strict for every $\lambda$. The lower bound was proven in [2] for the model (1.1) and in the general situation (2.2) in [11], and is based on an idea by C. Monthus [14]. We mention that (the analog of) the lower bound in (2.11) was recently proven in [15] and [4] to become optimal in the limit $\lambda \rightarrow \infty$ for the "reduced copolymer model" introduced in [2, Sec. 4] (this is a copolymer model where the disorder law $\mathbb{P}$ depends on the coupling parameter $\lambda$ ).

As already mentioned, much attention has been devoted to the slope of the critical curve at the origin, in short the "critical slope",

$$
\lim _{\lambda \searrow 0} \frac{h_{c}(\lambda)}{\lambda}
$$

Existence of such limit is not at all obvious (and indeed was proven [5] only in the case of the "standard copolymer model" (1.1)), but is expected to hold in general. While the proof in [5] was given in the case $\mathbb{P}\left(\omega_{1}= \pm 1\right)=1 / 2$, it was shown in [13] (by a much softer argument) that the results of [5] imply (always for the model (1.1)) that the slope exists and is the same in the Gaussian case we are considering here. Moreover, the critical slope is expected to be a function only of $\alpha$ and not of the full $K(\cdot)$, at least for $0<\alpha<1$, and to be independent of the choice of the disorder law $\mathbb{P}$, as long as the $\omega_{n}$ 's are IID, with finite exponential moments, centered and of variance 1 . In contrast, it is known that the critical curve $\lambda \mapsto h_{c}(\lambda)$ does in general depend on the details of $K(\cdot)$ (this follows from [3, Prop. 2.11]) and of course on the disorder law $\mathbb{P}$. The belief in the universality of the critical slope is supported by the result of [5] which, beyond proving that the limit (2.12) exists, identifies it with the critical slope of a continuous copolymer model, where the simple random walk $S$ is replaced by a Brownian motion, and the $\omega_{n}$ 's by a white noise. 
Until recently, nothing was known about the value of the critical slope, except for

$$
\frac{1}{1+\alpha} \leq \liminf _{\lambda \searrow 0} \frac{h_{c}(\lambda)}{\lambda} \leq \limsup _{\lambda \searrow 0} \frac{h_{c}(\lambda)}{\lambda} \leq 1
$$

which follows from (2.6) and from the lower bound in (2.11) (note that the strict upper bound (2.11) does not imply a strict upper bound on the slope). None of these bounds is believed to be optimal. In particular, as we mentioned in the introduction, for the standard copolymer model (1.1) numerical simulations [6] suggest a value around 0.83 for the slope. This situation was much improved in [3]: if $\alpha>1$, then [3, Ths. 2.9 and 2.10]

$$
\max \left(\frac{1}{\sqrt{1+\alpha}}, \frac{1}{2}\right) \leq \liminf _{\lambda \searrow 0} \frac{h_{c}(\lambda)}{\lambda} \leq \limsup _{\lambda \searrow 0} \frac{h_{c}(\lambda)}{\lambda}<1 .
$$

Note that $\alpha>1$ and $\alpha \leq 1$ are profoundly different situations: the inter-arrival times of the renewal process have finite mean in the former case and infinite mean in the latter. Moreover, it was proven in [3, Th. 2.10] that there exists $\alpha_{0}<1$ (which can be estimated to be around 0.65), such that if $\alpha \geq \alpha_{0}$

$$
\liminf _{\lambda \searrow 0} \frac{h_{c}(\lambda)}{\lambda}>\frac{1}{1+\alpha}
$$

Note that this does not cover the case of the standard copolymer model (1.1), for which $\alpha=1 / 2$.

\subsubsection{A new upper bound on the critical slope}

Our main result is that the upper bound in (2.13) is always strict:

Theorem 2.1. For every $\alpha>0$ there exists $\rho(\alpha) \in(1 /(1+\alpha), 1)$ such that, whenever $K(\cdot)$ satisfies (2.1),

$$
\underset{\lambda \searrow 0}{\limsup } \frac{h_{c}(\lambda)}{\lambda} \leq \rho(\alpha)
$$

It is interesting to note that the upper bound (2.16) depends only on the exponent $\alpha$ and not the details of $K(\cdot)$. This is coherent with the mentioned belief in universality of the slope.

The new idea which allows to go beyond the results of [3, Th. 2.10] is to bound above the fractional moments of $Z_{N, \omega}$ in two steps:

1. first we chop the system into blocks of size $k$, the correlation length of the annealed model, and we decompose $Z_{N, \omega}$ according to which of the blocks contain points of $\tau$

2. only at that point we apply the inequality (3.12), where each of the $a_{i}$ corresponds to one of the pieces into which the partition function has been decomposed.

Remark 2.2. Theorem 2.1 holds in the more general situation where $\omega$ is a sequence of IID random variables with finite exponential moments and normalized so that $\mathbb{E} \omega_{1}=0, \mathbb{E} \omega_{1}^{2}=1$. We state the result and give the proof only in the Gaussian case simply to keep technicalities at a minimum. The extension to the general disorder law can be obtained following the lines of [3, Sec. 4.4]. 


\section{Proof of Theorem 2.1 .}

Fix $\alpha>0,1 /(1+\alpha)<\gamma<1$ and define

$$
A_{N, \gamma}:=\mathbb{E}\left(Z_{N, \omega}^{\gamma}\right)
$$

The reason why we restrict to $\gamma>1 /(1+\alpha)$ will be clear after (3.34). From now on we take $h=\rho \lambda$, where the value of

$$
\rho:=\rho(\alpha, \gamma) \in(\gamma, 1)
$$

will be chosen close to 1 later. Let

$$
k:=\left\lfloor\frac{1}{\lambda^{2}(1-\rho)}\right\rfloor
$$

and note that, irrespective of how $\rho$ is chosen, $k$ can be made arbitrarily large choosing $\lambda$ small (which is no restriction since in Theorem 2.1 we are interested in the limit $\lambda \searrow 0$ ). One sees from (2.8) that, apart from an inessential factor $2, k$ is just the inverse of the annealed free energy, i.e.,

$$
k=\left\lfloor\frac{2}{\lim _{N \rightarrow \infty}(1 / N) \log \mathbb{E} Z_{N, \omega}}\right\rfloor .
$$

We will show that, if $\rho(\alpha, \gamma)$ is sufficiently close to 1 , there exists $\lambda_{0}:=\lambda_{0}(\gamma, K(\cdot))>0$ such that for $0<\lambda<\lambda_{0}$ there exists $c:=c(\gamma, \lambda, K(\cdot))<\infty$ such that

$$
A_{N, \gamma} \leq c[K(N / k)]^{\gamma}
$$

for every $N \in k \mathbb{N}$. In particular, by Jensen's inequality and the fact that the sequence $\left\{(1 / N) \mathbb{E} \log Z_{N, \omega}\right\}_{N}$ has a non-negative limit,

$$
\mathrm{F}(\lambda, \rho(\alpha, \gamma) \lambda)=\lim _{n \rightarrow \infty} \frac{1}{n k} \mathbb{E} \log Z_{n k, \omega} \leq \lim _{n \rightarrow \infty} \frac{1}{n k \gamma} \log A_{n k, \gamma}=0
$$

for $\lambda<\lambda_{0}$. This implies (2.16) with

$$
\rho(\alpha):=\inf _{1 /(1+\alpha)<\gamma<1} \rho(\alpha, \gamma)
$$

From now on we assume that $(N / k)$ is integer and we divide the interval $\{1, \ldots, N\}$ into blocks

$$
B_{i}:=\{(i-1) k+1,(i-1) k+2, \ldots, i k\} \text { with } i=1, \ldots, N / k .
$$

Set $Z_{i, j}:=Z_{(j-i), \theta^{i} \omega}$ (with the convention $Z_{i, i}=1$ ), where $\theta$ is the left shift operator: $\left(\theta^{a} \omega\right)_{i}:=$ $\omega_{i+a}$ for $i, a \in \mathbb{N}$. We have then the identity (see Fig. 1)

$$
Z_{N, \omega}=\sum_{\ell=1}^{N / k} \sum_{i_{0}:=0<i_{1}<\ldots<i_{\ell}=N / k} \widehat{Z}_{\omega}^{\left(i_{1}, \ldots, i_{\ell}\right)}
$$




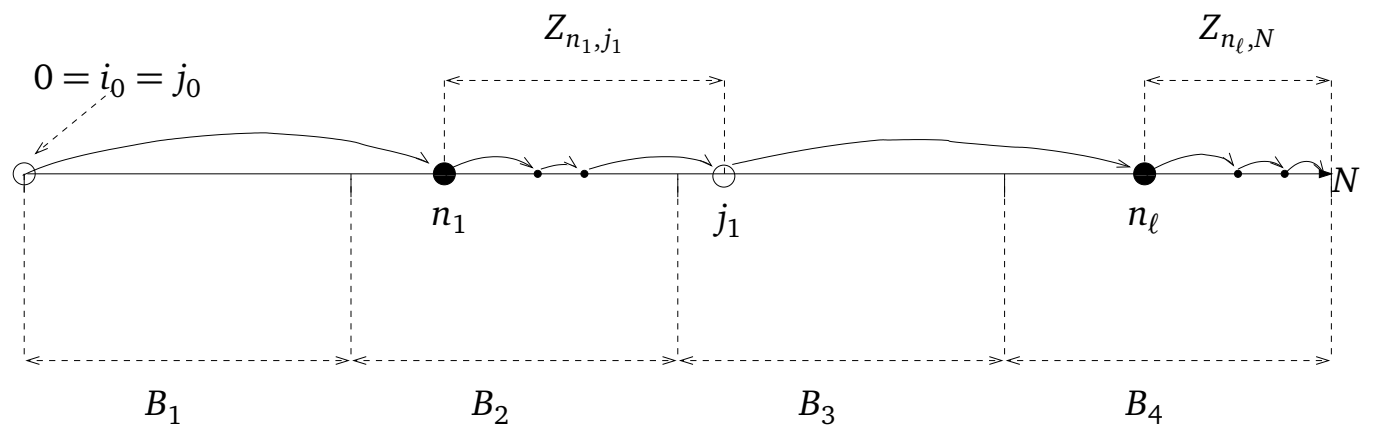

FIGURE 1. A typical configuration which contributes to $\widehat{Z}_{\omega}^{\left(i_{1}, \ldots, i_{\ell}\right)}$ defined in (3.10). In this example we have a number $N / k=4$ of blocks, $\ell=2$ and $i_{1}=2$, while $i_{\ell}=N / k=4$ by definition (cf. (3.9)). Big black dots denote the $n_{i}$ 's, white dots denote the $j_{i}$ 's, while small dots are all the other points of $\tau$. Note that $j_{1}-n_{1}<k$, as it should, and that there is no point of $\tau$ between a white dot and the next big black dot. In this example, the set $M$ of (3.14) is $\{2,3,4\}$, and as a consequence $W$ defined in (3.15) is $B_{2} \cup B_{3} \cup B_{4}$. In words: $n_{1}$ is the first point of $\tau$ after $0, j_{1}$ is the last point of $\tau$ which does not exceed $n_{1}+k-1, n_{2}$ is the first point after $j_{1}$, and so on. The index of the block containing $n_{r}$ defines $i_{r}$.

where

$$
\begin{aligned}
\widehat{Z}_{\omega}^{\left(i_{1}, \ldots, i_{\ell}\right):=} & \sum_{n_{1} \in B_{i_{1}}} \sum_{j_{1}=n_{1}}^{n_{1}+k-1} \sum_{\substack{n_{2} \in B_{i_{2}}: \\
n_{2} \geq n_{1}+k}} \sum_{j_{2}=n_{2}}^{n_{2}+k-1} \cdots \sum_{\substack{n_{\ell-1} \in B_{i_{\ell-1}} \\
n_{\ell-1} \geq n_{\ell-2}+k}} \sum_{j_{\ell-1}=n_{\ell-1}}^{n_{\ell-1}+k-1} \sum_{\substack{n_{\ell} \in B_{N / k}: \\
n_{\ell} \geq n_{\ell-1}+k}} \\
& \times \varphi\left(\left(0, n_{1}\right]\right) K\left(n_{1}\right) Z_{n_{1}, j_{1}} \varphi\left(\left(j_{1}, n_{2}\right]\right) K\left(n_{2}-j_{1}\right) Z_{n_{2}, j_{2}} \times \ldots \\
& \times \varphi\left(\left(j_{\ell-1}, n_{\ell}\right]\right) K\left(n_{\ell}-j_{\ell-1}\right) Z_{n_{\ell}, N}
\end{aligned}
$$

and, for $I \subset \mathbb{N}$,

$$
\varphi(I):=\frac{1+e^{-2 \lambda h|I|-2 \lambda \sum_{i \in I} \omega_{i}}}{2}
$$

We have then, using the inequality

$$
\left(\sum_{i=1}^{n} a_{i}\right)^{\gamma} \leq \sum_{i=1}^{n} a_{i}^{\gamma}
$$

which holds for $0 \leq \gamma \leq 1$ and $a_{i} \geq 0$,

$$
A_{N, \gamma} \leq \sum_{\ell=1}^{N / k} \sum_{i_{0}:=0<i_{1}<\ldots<i_{\ell}=N / k} \mathbb{E}\left[\left(\widehat{Z}_{\omega}^{\left(i_{1}, \ldots, i_{\ell}\right)}\right)^{\gamma}\right] .
$$

Define

$$
\begin{gathered}
M:=M\left(i_{1}, \ldots, i_{\ell}\right):=\left\{i_{1}, i_{2}, \ldots, i_{\ell}\right\} \cup\left\{i_{1}+1, i_{2}+1, \ldots, i_{\ell-1}+1\right\} \\
W:=W\left(i_{1}, \ldots, i_{\ell}\right):=\cup_{u \in M} B_{u}
\end{gathered}
$$


and note that $1 \leq|M|<2 \ell$. With the conventions of Fig. $1, W$ is the union of the blocks $B_{i}$ which either contain a big black dot or such that $B_{i-1}$ contains a big black dot. Note also that, for every $r$, the interval $\left[n_{r}, j_{r}\right]$ is a subset of $W$.

We want first of all to show that the $\varphi$ 's can be effectively replaced by constants. To this purpose, we use the inequality

$$
\varphi\left(\left(j_{r}, n_{r+1}\right]\right) \leq 2 \varphi\left(\left(j_{r}, n_{r+1}\right] \backslash W\right) \varphi\left(\left(j_{r}, n_{r+1}\right] \cap W\right)
$$

(with the convention that $\varphi(\emptyset)=1$ and $j_{0}:=0$ ), where $W$ was defined in (3.15). This is simply due to the fact that, if $I_{1}$ and $I_{2}$ are two disjoint subsets of $\mathbb{N}$, one has $\varphi\left(I_{1} \cup I_{2}\right) \leq 2 \varphi\left(I_{1}\right) \varphi\left(I_{2}\right)$.

We note that the two factors in the right-hand side of (3.16) are independent random variables. Moreover, since

$$
\left(j_{r}, n_{r+1}\right] \backslash W=\cup_{i: i_{r}+1<i<i_{r+1}} B_{i}
$$

we observe that the law of

$$
\prod_{r=0}^{\ell-1} \varphi\left(\left(j_{r}, n_{r+1}\right] \backslash W\right)
$$

depends only on $\left(i_{1}, \ldots, i_{\ell}\right)$ and not on the $n_{r}$ 's and $j_{r}$ 's, and that, once $\left(i_{1}, \ldots, i_{\ell}\right)$ is fixed, (3.18) is the product of $\ell$ independent random variables. As a consequence,

$$
A_{N, \gamma} \leq \sum_{\ell=1}^{N / k} \sum_{i_{0}:=0<i_{1}<\ldots<i_{\ell}=N / k} 2^{\gamma \ell} \prod_{r=0}^{\ell-1} \mathbb{E}\left(\varphi\left(\left(j_{r}, n_{r+1}\right] \backslash W\right)^{\gamma}\right) \mathbb{E}\left[\left(\check{Z}_{\omega}^{\left(i_{1}, \ldots, i_{\ell}\right)}\right)^{\gamma}\right],
$$

where

$$
\begin{aligned}
& \check{Z}_{\omega}^{\left(i_{1}, \ldots, i_{\ell}\right)}:=\sum_{n_{1} \in B_{i_{1}}} \sum_{j_{1}=n_{1}}^{n_{1}+k-1} \sum_{\substack{n_{2} \in B_{i_{2}}: \\
n_{2} \geq n_{1}+k}} \sum_{j_{2}=n_{2}}^{n_{2}+k-1} \cdots \sum_{\substack{n_{\ell-1} \in B_{i_{\ell-1}}: \\
n_{\ell-1} \geq n_{\ell-2}+k}}^{n_{\ell-1}+k-1} \sum_{j_{\ell-1}=n_{\ell-1}} \sum_{\substack{n_{\ell} \in B_{N} / k \\
n_{\ell} \geq n_{\ell-1}+k}} \\
& \times \varphi\left(\left(0, n_{1}\right] \cap W\right) K\left(n_{1}\right) Z_{n_{1}, j_{1}} \varphi\left(\left(j_{1}, n_{2}\right] \cap W\right) K\left(n_{2}-j_{1}\right) Z_{n_{2}, j_{2}} \times \ldots \\
& \times \varphi\left(\left(j_{\ell-1}, n_{\ell}\right] \cap W\right) K\left(n_{\ell}-j_{\ell-1}\right) Z_{n_{\ell}, N} .
\end{aligned}
$$

Thanks to (3.12) and to the choice $h=\rho \lambda$, for every $I \subset \mathbb{N}$

$$
\mathbb{E}\left(\varphi(I)^{\gamma}\right) \leq \frac{1+e^{2 \lambda^{2} \gamma(\gamma-\rho)|I|}}{2^{\gamma}} \leq 2^{1-\gamma},
$$

where the second inequality is implied by our assumption $\gamma<\rho$, cf. (3.2). Then,

$$
A_{N, \gamma} \leq \sum_{\ell=1}^{N / k} \sum_{i_{0}:=0<i_{1}<\ldots<i_{\ell}=N / k} 2^{\ell} \mathbb{E}\left[\left(\check{Z}_{\omega}^{\left(i_{1}, \ldots, i_{\ell}\right)}\right)^{\gamma}\right]
$$

In order to estimate the remaining average, we use Hölder's inequality with $p=1 / \gamma$ and $q=$ $1 /(1-\gamma)$ :

$$
\mathbb{E}\left[\left(\check{Z}_{\omega}^{\left(i_{1}, \ldots, i_{\ell}\right)}\right)^{\gamma}\right]=\widetilde{\mathbb{E}}\left[\left(\check{Z}_{\omega}^{\left(i_{1}, \ldots, i_{\ell}\right)}\right)^{\gamma} \frac{\mathrm{d} \mathbb{P}}{\mathrm{d} \widetilde{\mathbb{P}}}(\omega)\right] \leq\left(\widetilde{\mathbb{E}} \check{Z}_{\omega}^{\left(i_{1}, \ldots, i_{\ell}\right)}\right)^{\gamma}\left(\widetilde{\mathbb{E}}\left(\frac{\mathrm{d} \mathbb{P}}{\mathrm{d} \widetilde{\mathbb{P}}}\right)^{1 /(1-\gamma)}\right)^{1-\gamma}
$$


where, under the modified law $\widetilde{\mathbb{E}}:=\widetilde{\mathbb{E}}^{\left(i_{1}, \ldots, i_{\ell}\right)}$, the $\left\{\omega_{i}\right\}_{i \in \mathbb{N}}$ are still Gaussian, independent and of variance 1 , but $\omega_{i}$ has average $1 / \sqrt{k}$ if $i \in W\left(i_{1}, \ldots, i_{\ell}\right)$, while $\omega_{i}$ has average 0 , as under $\mathbb{E}$, if $i \notin W\left(i_{1}, \ldots, i_{\ell}\right)$. Since $\widetilde{\mathbb{E}}$ is still a product measure, it is immediate to check that

$$
\left(\widetilde{\mathbb{E}}\left(\frac{\mathrm{d} \mathbb{P}}{\mathrm{d} \widetilde{\mathbb{P}}}\right)^{1 /(1-\gamma)}\right)^{1-\gamma}=\left[\mathbb{E} e^{\left(\omega_{1} / \sqrt{k}+1 /(2 k)\right) \frac{\gamma}{1-\gamma}}\right]^{(1-\gamma)|W|}=e^{\frac{\gamma|W|}{2 k(1-\gamma)}} \leq e^{\frac{\gamma}{1-\gamma} \ell},
$$

where we used the fact that $|W|=k|M|$ and $|M| \leq 2 \ell$. Next, we observe that

$$
\widetilde{\mathbb{E}} \varphi\left(\left(j_{r}, n_{r+1}\right] \cap W\right)=\frac{1+\widetilde{\mathbb{E}} e^{-2 \lambda h|I|-2 \lambda \sum_{i \in I} \omega_{i}}}{2}
$$

with $I=\left(j_{r}, n_{r+1}\right] \cap W$. Thanks to the definition of $k$ and to $h=\rho \lambda$, (3.25) equals

$$
\frac{1+e^{2 \lambda^{2}|I|(1-\rho)-2 \lambda|I| / \sqrt{k}}}{2} \leq \frac{1+e^{2 \lambda^{2}|I|(1-\rho)-2|I| \lambda^{2} \sqrt{1-\rho}}}{2} \leq 1 .
$$

In conclusion, we proved

$$
\begin{aligned}
A_{N, \gamma} & \leq \sum_{\ell=1}^{N / k} \sum_{i_{0}:=0<i_{1}<\ldots<i_{\ell}=N / k}\left(2 e^{\frac{\gamma}{1-\gamma}}\right)^{\ell} \\
& \times\left[\sum_{n_{1} \in B_{i_{1}}} \ldots \sum_{\substack{n_{\ell} \in B_{N / k}: \\
n_{\ell} \geq n_{\ell-1}+k}} K\left(n_{1}\right) \ldots K\left(n_{\ell}-j_{\ell-1}\right) U\left(j_{1}-n_{1}\right) \ldots U\left(N-n_{\ell}\right)\right]^{\gamma}
\end{aligned}
$$

where, since $\left[n_{r}, j_{r}\right]$ is a subset of $W$ as observed above,

$$
U(n):=\mathbb{E} Z_{n, \omega}(\lambda, \rho \lambda+1 / \sqrt{k})
$$

with the convention that $U(0):=1$. In $(3.28)$ we used independence of $Z_{n_{r}, j_{r}}$ for different $r$ 's (recall that $\widetilde{\mathbb{E}}$ is a product measure) to factorize the expectation. The heart of the proof of Theorem 2.1 is the following:

Lemma 3.1. There exists $\lambda_{0}(\gamma, K(\cdot))>0$ such that the following holds for $\lambda<\lambda_{0}$. If, for some $\varepsilon>0$,

$$
\sum_{j=0}^{k-1} U(j) \leq \varepsilon Y\left(k, \mathscr{C}_{K}, \alpha\right):=\varepsilon \times\left\{\begin{array}{lll}
\frac{k^{\alpha}}{\mathscr{C}_{K}} & \text { if } & \alpha<1 \\
\frac{2 k}{\mathscr{C}_{K} \log k} & \text { if } & \alpha=1 \\
k & \text { if } & \alpha>1
\end{array}\right.
$$

and

$$
\sum_{j=0}^{k-1} \sum_{n \geq k} U(j) K(n-j) \leq \varepsilon
$$


then the quantity in square brackets in (3.28) is bounded above by

$$
C_{1} \varepsilon^{\ell} 3^{(3+2 \alpha) \ell} \prod_{r=1}^{\ell} \frac{1}{\left(i_{r}-i_{r-1}\right)^{1+\alpha}}
$$

where $C_{1}:=C_{1}(\varepsilon, k, K(\cdot))<\infty$.

Here and in the following, the positive and finite constants $C_{i}, i \geq 1$ depend only on the arguments which are explicitly indicated, while $\mathscr{C}_{K}$ is the same constant which appears in (2.1).

Assume that Lemma 3.1 is true and that (3.30)-(3.31) are satisfied. Then,

$$
\begin{aligned}
A_{N, \gamma} \leq C_{1}(\varepsilon, k, K(\cdot))^{\gamma} \sum_{\ell=1}^{N / k} \sum_{i_{0}:=0<i_{1}<\ldots<i_{\ell}=N / k} & \left(3^{\gamma(3+2 \alpha)} 2 e^{\frac{\gamma}{1-\gamma}}\right)^{\ell} \varepsilon^{\gamma \ell} \\
& \times \prod_{r=1}^{\ell} \frac{1}{\left(i_{r}-i_{r-1}\right)^{(1+\alpha) \gamma}} .
\end{aligned}
$$

If moreover $\varepsilon$ satisfies

$$
\xi:=3^{\gamma(3+2 \alpha)} 2 e^{\frac{\gamma}{1-\gamma}} \varepsilon^{\gamma} \sum_{n \in \mathbb{N}} \frac{1}{n^{(1+\alpha) \gamma}}<1
$$

then it follows from [11, Th. A.4] that

$$
A_{N, \gamma} \leq C_{2}(\varepsilon, \gamma, k, K(\cdot))(N / k)^{-(1+\alpha) \gamma}
$$

for every $N \in k \mathbb{N}$. Indeed, the sum in the right-hand side of (3.33) is nothing but the partition function of a homogeneous pinning model [11, Ch. 2] of length $N / k$ with pinning parameter $\xi$ such that the system is in the delocalized phase (this is encoded in (3.34)). More precisely: to obtain (3.35) it is sufficient to apply Proposition 3.2 below, with $\alpha$ replaced by $(1+\alpha) \gamma-1>0$ and $K(\cdot)$ replaced by

$$
\widehat{K}(n)=\frac{1}{n^{(1+\alpha) \gamma}}\left(\sum_{j \in \mathbb{N}} \frac{1}{j^{(1+\alpha) \gamma}}\right)^{-1} .
$$

Proposition 3.2. [11, Th. A.4] If $K(\cdot)$ is a probability on $\mathbb{N}$ which satisfies (2.1) for some $\alpha>0$, then for every $\xi<1$ there exists $c=c(K(\cdot), \xi)$ such that for every $N \in \mathbb{N}$

$$
\sum_{\ell=1}^{N} \sum_{0=i_{0}<\ldots<i_{\ell}=N} \xi^{\ell} \prod_{r=1}^{\ell} K\left(i_{r}-i_{r-1}\right) \leq c K(N) .
$$

Inequality (3.5) is then proven; note that $C_{2}$ depends on $\lambda$ through $k$. The condition $\gamma>1 /(1+\alpha)$ which we required since the beginning guarantees that the sum in (3.34) converges. Note that (3.34) depends on $K(\cdot)$ only through $\alpha$; this is important since we want $\rho$ in (2.16) to depend only on $\alpha$ and not on the whole $K(\cdot)$. 
To conclude the proof of Theorem 2.1, we still have to prove Lemma 3.1 and to show that (3.30)(3.31) can be satisfied, with $\varepsilon:=\varepsilon(\alpha, \gamma)$ satisfying (3.34), for all $\lambda \leq \lambda_{0}$ and $h=\lambda \rho(\alpha, \gamma)$, if $\lambda_{0}(\gamma, K(\cdot))$ is sufficiently small and $\rho(\alpha, \gamma)$ is close to 1 (see also Remark 3.5 below).

Proof of Lemma 3.1. First of all, we get rid of $U\left(N-n_{\ell}\right)$ and effectively we replace $n_{\ell}$ by $N$ : the quantity in square brackets in (3.28) is upper bounded by

$$
\begin{array}{rl}
C_{3}(k, K(\cdot)) \sum_{n_{1} \in B_{i_{1}}} \ldots \sum_{j_{\ell-1}=n_{\ell-1}}^{n_{\ell-1}+k-1} & K\left(n_{1}\right) \ldots K\left(n_{\ell-1}-j_{\ell-2}\right) K\left(N-j_{\ell-1}\right) \\
& \times U\left(j_{1}-n_{1}\right) \ldots U\left(j_{\ell-1}-n_{\ell-1}\right) .
\end{array}
$$

Explicitly, one may take

$$
C_{3}(k, K(\cdot))=k U\left(N-n_{\ell}\right) \sup _{\substack{j, n, N: \\ 0<j<n \\ N-k \leq n \leq N}} \frac{K(n-j)}{K(N-j)}:
$$

one has

$$
U\left(N-n_{\ell}\right) \leq \mathbb{E} Z_{N-n_{\ell}, \omega}(\lambda, \rho \lambda) \leq e^{2 \lambda^{2} k}
$$

(recall (2.9) and $N-n_{\ell} \leq k$ ), while the supremum in (3.39) is easily seen from (2.1) to depend only on $k$ and $K(\cdot)$.

Recall that by convention $i_{0}=j_{0}=0$, and let also from now on $n_{\ell}:=N$ and $n_{0}:=0$. We do the following:

- for every $1 \leq r \leq \ell$ such that $i_{r}>i_{r-1}+2$ (which guarantees that between $j_{r-1}$ and $n_{r}$ there is at least one full block), we use

$$
K\left(n_{r}-j_{r-1}\right) \leq 3^{\alpha+2} \frac{\mathscr{C}_{K}}{\left(i_{r}-i_{r-1}\right)^{1+\alpha} k^{1+\alpha}} .
$$

This is true under the assumption that $k \geq k_{0}$ with $k_{0}(\gamma, K(\cdot))$ large enough, i.e., $\lambda \leq$ $\lambda_{0}(\gamma, K(\cdot))$ with $\lambda_{0}$ small, since $n_{r}-j_{r-1} \geq k\left(i_{r}-i_{r-1}-2\right)$ and $\sup _{n>2}(n /(n-2))^{1+\alpha}=3^{1+\alpha}$.

- for every $1 \leq r \leq \ell$ such that $i_{r} \leq i_{r-1}+2$, we leave $K\left(n_{r}-j_{r-1}\right)$ as it is.

Then, (3.38) is bounded above by

$$
\begin{aligned}
& C_{3}(k, K(\cdot)) \frac{3^{(\alpha+2) \ell}}{k^{(1+\alpha)|J|}} \prod_{r \in J} \frac{\mathscr{C}_{K}}{\left(i_{r}-i_{r-1}\right)^{(1+\alpha)}} \\
& \times \sum_{n_{1} \in B_{i_{1}}} \ldots \sum_{j_{\ell-1}=n_{\ell-1}}^{n_{\ell-1}+k-1}\left(\prod_{r \in\{1, \ldots, \ell\} \backslash J} K\left(n_{r}-j_{r-1}\right)\right) U\left(j_{1}-n_{1}\right) \ldots U\left(j_{\ell-1}-n_{\ell-1}\right),
\end{aligned}
$$

where

$$
J:=J\left(i_{1}, \ldots, i_{\ell}\right):=\left\{1 \leq r \leq \ell: i_{r}>i_{r-1}+2\right\} .
$$

Now we can sum over $j_{r}, n_{r}, 1 \leq r<\ell$, using the two assumptions (3.30)-(3.31). We do this in three steps: 
- First, for every $r \in J$ we sum over the allowed values of $j_{r-1}$ (provided that $r>1$, otherwise there is no $j_{r-1}$ to sum over) using (3.30) and the constraint $0 \leq j_{r-1}-n_{r-1}<k$. The sum over all such $j_{r-1}$ gives at most

$$
C_{4}(\varepsilon, k, K(\cdot)) \varepsilon^{|J|} Y\left(k, \mathscr{C}_{K}, \alpha\right)^{|J|}
$$

where $C_{4}$ takes care of the fact that possibly $1 \in J$. At this point we are left with the task of summing

$$
\prod_{r \in\{1, \ldots, \ell\} \backslash J}\left[K\left(n_{r}-j_{r-1}\right) U\left(j_{r-1}-n_{r-1}\right)\right]
$$

(observe that $U\left(j_{0}-n_{0}\right)=1$ ) over all the allowed values of $n_{r}, 1 \leq r<\ell$ and of $j_{r-1}, r \in$ $\{1, \ldots, \ell\} \backslash J$.

- Secondly, using (3.31), we see that if $r \in\{1, \ldots, \ell\} \backslash J$ then the sum of $K\left(n_{r}-j_{r-1}\right) U\left(j_{r-1}-\right.$ $n_{r-1}$ ) over the allowed values of $n_{r}$ and $j_{r-1}$ gives at most $\varepsilon$ (or 1 if $r=1$ ). The contribution from all the $n_{r}, j_{r-1}$ with $r \in\{1, \ldots, \ell\} \backslash J$ is therefore at most

$$
C_{5}(\varepsilon, k, K(\cdot)) \varepsilon^{\ell-|J|} .
$$

This is best seen if one starts to sum on $n_{r}, j_{r-1}$ for the largest value of $r \in\{1, \ldots, \ell\} \backslash J$ and then proceeds to the second largest, and so on.

- Finally, the sum over all the $n_{r}$ 's with $r \in J$ is trivial (the summand does not depend on the $n_{r}$ 's) and gives at most $k^{|J|}$.

In conclusion, we have upper bounded (3.41) by

$$
C_{6}(\varepsilon, k, K(\cdot)) 3^{(3+2 \alpha) \ell} \varepsilon^{\ell} \prod_{r=1}^{\ell} \frac{1}{\left(i_{r}-i_{r-1}\right)^{1+\alpha}}\left[\frac{\mathscr{C}_{K} Y\left(k, \mathscr{C}_{K}, \alpha\right)}{k^{\alpha}}\right]^{|J|} .
$$

If $0<\alpha<1$ it is clear from the definition of $Y\left(k, \mathscr{C}_{K}, \alpha\right)$ that the last factor equals 1 and (3.32) is proven. For $\alpha \geq 1$,

$$
\frac{\mathscr{C}_{K} Y\left(k, \mathscr{C}_{K}, \alpha\right)}{k^{\alpha}}
$$

can be made as small as wished with $k$ large (i.e. choosing $\lambda_{0}$ small), so that we can again assume that the last factor in (3.46) does not exceed 1. Lemma 3.1 is proven.

Finally, we have:

Proposition 3.3. Let $\lambda_{0}(\gamma, K(\cdot))>0$ be sufficiently small. There exists $\varepsilon:=\varepsilon(\alpha, \gamma)>0$ satisfying (3.34) and $\rho:=\rho(\alpha, \gamma) \in(\gamma, 1)$ such that conditions (3.30)-(3.31) are satisfied for all $0<\lambda<\lambda_{0}$ and $h=\rho \lambda$. 
Proof of Proposition 3.3. We have by direct computation

$$
\begin{aligned}
U(j) & =\mathbf{E}\left[\prod_{1 \leq n: \tau_{n} \leq j} \frac{1+e^{\left(2 \lambda^{2}(1-\rho)-2 \lambda / \sqrt{k}\right)\left(\tau_{n}-\tau_{n-1}\right)}}{2} \mathbf{1}_{\{j \in \tau\}}\right] \\
& \leq \mathbf{E}\left[\prod_{1 \leq n: \tau_{n} \leq j} \frac{1+e^{-\frac{1}{k \sqrt{1-\rho}}\left(\tau_{n}-\tau_{n-1}\right)}}{2} \mathbf{1}_{\{j \in \tau\}}\right] \\
& =\mathbf{P}(j \in \tau) \mathbf{E}\left[\prod_{1 \leq n: \tau_{n} \leq j} \frac{1+e^{-\frac{1}{k \sqrt{1-\rho}}\left(\tau_{n}-\tau_{n-1}\right)}}{2} \mid j \in \tau\right]
\end{aligned}
$$

where in the inequality we assume that $2 \sqrt{1-\rho}<1$ (it is important that this condition does not depend on $\lambda$ ). Of course, from (3.47) we see that for every $j$

$$
U(j) \leq \mathbf{P}(j \in \tau)
$$

Moreover, we know from [8, Th. B] that, if $0<\alpha<1$,

$$
\mathbf{P}(j \in \tau) \stackrel{j \rightarrow \infty}{\sim} \frac{\alpha \sin (\pi \alpha)}{\pi} \frac{1}{\mathscr{C}_{K} j^{1-\alpha}},
$$

while for $\alpha=1$

$$
\mathbf{P}(j \in \tau) \stackrel{j \rightarrow \infty}{\sim} \frac{1}{\mathscr{C}_{K} \log j}
$$

(cf. for instance [11, Th. A.6]). For every $a \in(0,1)$ one has then for $k$ sufficiently large, i.e., for $\lambda_{0}$ small,

$$
\sum_{j=0}^{a k} U(j) \leq \sum_{j=0}^{a k} \mathbf{P}(j \in \tau) \leq Y\left(a k, \mathscr{C}_{K}, \alpha\right)
$$

(we recall that $Y(\cdot, \cdot, \cdot)$ was defined in (3.30)). We need also the following fact:

Lemma 3.4. For every $\alpha>0$ there exists $C_{7}(\alpha)<\infty$ such that the following holds. If $0<\alpha<1$ then, say, for $q>2$

$$
\limsup _{N \rightarrow \infty} \mathbf{E}\left[\prod_{1 \leq j: \tau_{j} \leq N} \frac{1+e^{-(q / N)\left(\tau_{j}-\tau_{j-1}\right)}}{2} \mid N \in \tau\right] \leq C_{7}(\alpha) \frac{(\log q)^{2}}{q^{\alpha}} .
$$

If $\alpha \geq 1$ then, for every $q>0$,

$$
\limsup _{N \rightarrow \infty} \mathbf{E}\left[\prod_{1 \leq j: \tau_{j} \leq N} \frac{1+e^{-(q / N)\left(\tau_{j}-\tau_{j-1}\right)}}{2} \mid N \in \tau\right] \leq C_{7}(\alpha) e^{-q / 2} .
$$


Lemma 3.4 was proven in [3, Lemma 4.3]. We add a few side remarks about its proof in Appendix A.

The upper bound (3.53) is certainly not optimal, but it gives us an estimate which vanishes for $q \rightarrow \infty$ and which depends only on $\alpha$ and $q$, which is all we need in the following. Let us mention that in the case of the standard copolymer model, using the property that for every $N \in 2 \mathbb{N}$ and every $k \in\{0,2, \ldots, N\}$

$$
\mathbf{P}^{\mathrm{SRW}}\left[\left|\left\{1 \leq n \leq N: \operatorname{sign}\left(S_{n}\right)=-1\right\}\right|=k \mid N \in \tau\right]=\frac{1}{(N / 2)+1}
$$

(see [9, Ch. III.9]) we obtain for every $q>0$

$$
\lim _{N \rightarrow \infty} \mathbf{E}^{\mathrm{SRW}}\left[\prod_{1 \leq j: \tau_{j} \leq N} \frac{1+e^{-(q / N)\left(\tau_{j}-\tau_{j-1}\right)}}{2} \mid N \in \tau\right]=\frac{1-e^{-q}}{q} .
$$

\subsubsection{Proof of (3.30)}

Fix $\varepsilon:=\varepsilon(\alpha, \gamma)$ which satisfies (3.34) and choose

$$
a:=a(\varepsilon, \alpha):=\left\{\begin{array}{lll}
(\varepsilon / 2)^{1 / \alpha} & \text { if } & 0<\alpha<1 \\
\varepsilon / 4 & \text { if } \quad \alpha=1 \\
\varepsilon / 2 & \text { if } \quad \alpha>1 .
\end{array}\right.
$$

Via (3.52) one finds (for $k$ sufficiently large)

$$
\sum_{j=0}^{a k} U(j) \leq \frac{\varepsilon}{2} Y\left(k, \mathscr{C}_{K}, \alpha\right)
$$

Next we observe that, for $a k \leq j<k$, choosing $\lambda_{0}$ small and $\rho$ sufficiently close to 1 (how close, depending on $K(\cdot)$ only through the exponent $\alpha$ ) we have $U(j) \leq(\varepsilon / 2) \mathbf{P}(j \in \tau)$. This just follows from Lemma 3.4 above (applied with $N \sim a k$ and $q=a / \sqrt{1-\rho}$ ) and from (3.48), since $\lambda_{0}$ small implies $k$ large. As a consequence,

$$
\sum_{j=a k}^{k-1} U(j) \leq \frac{\varepsilon}{2} Y\left(k, \mathscr{C}_{K}, \alpha\right)
$$

and (3.30) follows.

Remark 3.5. It is probably useful to summarize the logic of the proof of (3.30) (similar observations hold for the proof of (3.31) below). Given $\alpha$, one first fixes $1 /(1+\alpha)<\gamma<1$, then $\varepsilon(\alpha, \gamma)$ which satisfies (3.34), then $a=a(\varepsilon, \alpha)$ as in (3.57) and $\rho=\rho(\alpha, \gamma)<1$ such that the right-hand side of Eqs. (3.53)-(3.54) is smaller than $(\varepsilon / 2)$ when $q$ is replaced by $a / \sqrt{1-\rho}$. Once all these parameters are fixed, one chooses $\lambda_{0}$ sufficiently small (i.e. $k$ sufficiently large) so that for all $\lambda<\lambda_{0}, h=\rho \lambda$ the estimates (3.58)-(3.59) hold. 


\subsubsection{Proof of (3.31)}

If we choose $b:=b(\varepsilon, \alpha)$ small, $j \leq b k$ implies $k-j \geq k / 2$. Therefore,

$$
\sum_{j=0}^{b k} \sum_{n \geq k} U(j) K(n-j) \leq C_{8}(\alpha) \frac{C_{K}}{k^{\alpha}} \sum_{j=0}^{b k} \mathbf{P}(j \in \tau) \leq \frac{\varepsilon}{2}
$$

(if $k$ is sufficiently large and $b(\varepsilon, \alpha)$ is suitably small). As for the rest of the sum: again from Lemma 3.4 and (3.48), one has $U(j) \leq(\varepsilon / 2) \mathbf{P}(j \in \tau)$ for every $b k \leq j<k$. Then,

$$
\sum_{j=b k}^{k-1} \sum_{n \geq k} U(j) K(n-j) \leq \frac{\varepsilon}{2} \sum_{j=0}^{k-1} \sum_{n \geq k} \mathbf{P}(j \in \tau) K(n-j)=\frac{\varepsilon}{2}
$$

In the last equality, we used the fact that

$$
\sum_{j=0}^{k-1} \mathbf{P}(j \in \tau) K(n-j)
$$

is the P-probability that the first point of $\tau$ which does not precede $k$ equals $n$. The sum over $n \geq k$ of (3.62) then clearly equals 1 , since $\tau$ is recurrent.

\section{A Remarks on the proof of Lemma 3.4 .}

The proof of Lemma 3.4 given in [3, Lemma 4.3] works as follows. Let $X_{N}:=\max \{n=0, \ldots, N$ : $n \in \tau$ \}, i.e., the last point of $\tau$ up to $N$. First of all one shows that

$$
\begin{aligned}
& \limsup _{N \rightarrow \infty} \mathbf{E}\left[\prod_{1 \leq j: \tau_{j} \leq N} \frac{1+e^{-(q / N)\left(\tau_{j}-\tau_{j-1}\right)}}{2} \mid N \in \tau\right] \\
& \leq C_{9}(\alpha) \limsup _{N \rightarrow \infty} \mathrm{E}\left[\frac{1+e^{-(q / N)\left(N-X_{N}\right)}}{2} \prod_{1 \leq j: \tau_{j} \leq N} \frac{1+e^{-(q / N)\left(\tau_{j}-\tau_{j-1}\right)}}{2}\right] \text {. }
\end{aligned}
$$

We detail below the proof of this inequality in order to leave no doubts on the fact that the constant $C_{9}$ depends only on $\alpha$. This was not emphasized in the proof of [3, Lemma 4.3] since it was not needed there.

For $\alpha \geq 1$, it follows from [3, Eqs. (4.23) and (4.49)] that the lim sup in the right-hand side of (A.2) is actually a limit, and equals $\exp (-q / 2)$ (the $q / 8$ which appears in [3, Eq. (4.49)] can be immediately improved into $q / 2$ ). As a side remark, the expectation in (A.2), irrespective of the value of $\alpha$ and $N$, is not smaller than $\exp (-q / 2)$; this just follows from the convexity of the exponential function:

$$
\frac{1+e^{-(q / N) x}}{2} \geq e^{-q /(2 N) x} .
$$

For $0<\alpha<1$, the limsup in (A.2) does not exceed $C_{10}(\alpha)(\log q)^{2} / q^{\alpha}$, as was proven in [3, Eq. (4.43)]. 
Finally we prove (A.2), which is quite standard. The expectation in (A.1) is bounded above by

$$
\begin{aligned}
& \mathbf{E}\left[\frac{1+e^{-(q / N)\left(N / 2-X_{N / 2}\right)}}{2} \prod_{1 \leq j: \tau_{j} \leq N / 2} \frac{1+e^{-(q / N)\left(\tau_{j}-\tau_{j-1}\right)}}{2} \mid N \in \tau\right] \\
& =\sum_{i=0}^{N / 2} \mathbf{E}\left[\frac{1+e^{-(q / N)(N / 2-i)}}{2} \prod_{1 \leq j: \tau_{j} \leq N / 2} \frac{1+e^{-(q / N)\left(\tau_{j}-\tau_{j-1}\right)}}{2} \mid X_{N / 2}=i\right] \\
& \times \mathbf{P}\left(X_{N / 2}=i \mid N \in \tau\right)
\end{aligned}
$$

and (A.2) follows if we can prove that

$$
\limsup _{N \rightarrow \infty} \max _{0 \leq i \leq N / 2} \frac{\mathbf{P}\left(X_{N / 2}=i \mid N \in \tau\right)}{\mathbf{P}\left(X_{N / 2}=i\right)} \leq C_{11}(\alpha) .
$$

To show this, we use repeatedly (3.50) and (2.1). We start from the identity

$$
\frac{\mathbf{P}\left(X_{N / 2}=i \mid N \in \tau\right)}{\mathbf{P}\left(X_{N / 2}=i\right)}=\frac{\sum_{j=(N / 2)+1}^{N} K(j-i) \mathbf{P}(N-j \in \tau)}{\mathbf{P}(N \in \tau) \sum_{j=(N / 2)+1}^{\infty} K(j-i)}
$$

The denominator is lower bounded, uniformly in $0 \leq i \leq N / 2$, by

$$
\mathbf{P}(N \in \tau) \sum_{j=(N / 2)+1}^{\infty} K(j) \geq \frac{C_{12}(\alpha)}{N}
$$

where the last inequality holds for $N$ sufficiently large. As for the numerator: always for $N$ sufficiently large,

$$
\sum_{j=(N / 2)+1}^{(3 / 4) N} K(j-i) \mathbf{P}(N-j \in \tau) \leq C_{13} \mathbf{P}(N \in \tau) \sum_{j=(N / 2)+1}^{\infty} K(j-i)
$$

and, uniformly on $0 \leq i \leq N / 2$,

$$
\sum_{j=(3 / 4) N}^{N} K(j-i) \mathbf{P}(N-j \in \tau) \leq C_{14} \frac{\mathscr{C}_{K}}{N^{1+\alpha}} \sum_{j=0}^{N} \mathbf{P}(j \in \tau) \leq \frac{C_{15}(\alpha)}{N} .
$$

\section{Acknowledgments}

This work was partially supported by ANR, grant POLINTBIO and grant LHMSHE. I wish to thank the anonymous referees for the careful reading of the manuscript and for several useful comments. 


\section{References}

[1] K. S. Alexander and N. Zygouras, Quenched and annealed critical points in polymer pinning models, arXiv:0805.1708.

[2] T. Bodineau and G. Giacomin, On the localization transition of random copolymers near selective interfaces, J. Statist. Phys. 117 (2004), 801-818. MR2107896

[3] T. Bodineau, G. Giacomin, H. Lacoin and F. L. Toninelli, Copolymers at selective interfaces: new bounds on the phase diagram, J. Statist. Phys. 132 (2008), 603-626. MR2429695

[4] E. Bolthausen, F. Caravenna and B. de Tilière, The quenched critical point of a diluted disordered polymer model, Stochastic Process. Appl., to appear. arXiv:0711.0141v1 [math.PR].

[5] E. Bolthausen and F. den Hollander, Localization transition for a polymer near an interface, Ann. Probab. 25 (1997), 1334-1366. MR1457622

[6] F. Caravenna, G. Giacomin and M. Gubinelli, A Numerical Approach to Copolymers at Selective Interfaces, J. Statist. Phys. 122 (2006), 799-832. MR2213950

[7] B. Derrida, G. Giacomin, H. Lacoin and F. L. Toninelli, Fractional moment bounds and disorder relevance for pinning models, Commun. Math. Phys., to appear. arXiv:0712.2515 [math.PR].

[8] R. A. Doney, One-sided local large deviations and renewal theorems in the case of infinite mean, Probab. Theory Rel. Fields 107 (1997), 451-465. MR1440141

[9] W. Feller, An introduction to probability theory and its applications, Vol. I, Second Edition, John Wiley \& Sons, 1966. MR0210154

[10] T. Garel, D. A. Huse, S. Leibler and H. Orland, Localization transition of random chains at interfaces, Europhys. Lett. 8 (1989), 9-13.

[11] G. Giacomin, Random Polymer Models, Imperial College Press, World Scientific (2007). MR2380992

[12] G. Giacomin, H. Lacoin and F. L. Toninelli, Hierarchical pinning models, quadratic maps and quenched disorder, Probab. Theory Rel. Fields, to appear. arXiv:0711.4649 [math.PR].

[13] G. Giacomin and F. L. Toninelli, Estimates on path delocalization for copolymers at selective interfaces, Probab.Theory Rel. Fields 133 (2005), 464-482. MR2197110

[14] C. Monthus, On the localization of random heteropolymers at the interface between two selective solvents, Eur. Phys. J. B 13 (2000), 111-130.

[15] F. L. Toninelli, Disordered pinning models and copolymers: beyond annealed bounds, Ann. Appl. Probab. 18 (2008), 1569-1587. MR2434181 